\title{
SuperNova neutrino physics with nuclear emulsion detectors
}

\author{
Valerio Gentile* \\ Gran Sasso Science Institute - Viale F. Crispi, 767100 L'Aquila, Italy \\ E-mail: valerio.gentileegsi.it
}

\section{Giovanni De Lellis}

Dipartimento di Fisica dell'Università Federico II di Napoli, I-80125 Napoli, Italy

INFN Sezione di Napoli, 80125 Napoli, Italy

\author{
Antonia Di Crescenzo \\ Dipartimento di Fisica dell'Università Federico II di Napoli, I-80125 Napoli, Italy \\ INFN Sezione di Napoli, 80125 Napoli, Italy
}

\section{Andrea Gallo Rosso}

Gran Sasso Science Institute - Viale F. Crispi, 767100 L'Aquila, Italy

\section{Francesco Vissani}

INFN Laboratori Nazionali del Gran Sasso, Assergi (AQ), Italy

\begin{abstract}
Supernova explosions are very powerful phenomena occurring in the space. Although the observation of these events is very rarely achieved, the new generation of ton-scale detectors for dark matter search are sensitive to neutrinos coming for supernovae which can also represent a serious and unremoveable background source. A directional detector based on nuclear emulsions provides the unique capability to observe neutral current interactions of neutrinos originated by a supernova explosion. NEWSdm is a detector based on nuclear emulsions made of nanometric size crystals which can make the reconstruction of trajectories with path lengths down to $50 \mathrm{~nm}$ possible if analyzed by means of microscopes with enough resolution.
\end{abstract}

XVII International Workshop on Neutrino Telescope,

13-17 March 2017

Venice, Italy

${ }^{*}$ Speaker. 


\section{Introduction}

The explosion of a Supernova, due to the collapse of a star at the end of its life, is one of the most energetic observable phenomena in the Universe. Neutrinos are powerful messangers of a supernova explosion since they carry almost the total energy emitted by the source and are weakly interacting [1]. Therefore, they could directly explain the underlying processes inside a star leading to the the violent explosion. The energy spectrum of emitted neutrinos can be parametrized by a Maxwell-Boltzmann distribution [2] with the mean energies for each type of neutrinos provided by several models [1]. The total energy released by the supernova is the neutron star gravitational binding energy that is about $10^{53} \mathrm{erg}[1]$ corresponding to $\sim 10^{57}$ emitted neutrinos. Since the main processes leading to the neutrino emission are mediated by neutral current interaction we can assume the energy equipartition for each neutrinos and antineutrinos, as predicted also by several simulations [3, 4].

\section{Neutrino interaction with the detector}

The number of neutrinos interacting in a detector depends on the number of targets, the crosssection $\sigma(E)$ and the fluence $\mathscr{F}(E)$ of the incoming neutrinos:

$$
N(E)=\frac{d N}{d E}=\frac{M_{\text {riv }}}{A m_{\text {uma }}} \sigma(E) \mathscr{F}(E)
$$

where $M_{r i v}$ is the detector mass, $A$ is the atomic mass number, $m_{\text {uma }}$ is the atomic mass unit. Since the fluence has an inverse square dependence on distance $D$ between the Earth and the supernova explosion, the number of incoming neutrinos strongly depends on how far the source is located from the Earth. In the galactic centre the density of stars is very high and a supernova explosion is more likely to occur; a distance $D=10 \mathrm{Kpc}$ is therefore assumed in the present study. We focus on the coherent elastic neutrino nucleus scattering ( $\mathrm{CEvNS}$ ) correspondig to the following differential cross-section [5]:

$$
\frac{d \sigma}{d \Omega}=\frac{G_{F}^{2}}{(2 \pi)^{2}} \frac{Q_{W}^{2}}{4} E_{v}^{2}(1+\cos \theta) F^{2}(q)
$$

where $d \Omega$ is the solid angle, $G_{F}^{2}$ the Fermi constant, $Q_{w}$ the weak charge, $\theta$ the neutrino scattering angle and $F(q)$ the form factor assuming the Helm's model.

\section{NEWSdm detector}

NEWSdm (Nuclear emulsion for WIMP search with directional measurament) [6] is an experiment that foresees the use of nuclear emulsion both as target and tracking detector with nanometric resolution. The detector is surrounded by a shielding from environmental and cosmogenic background source suppression and it will be placed on an equatorial telescope in order to keep its orientation towards Cygnus constellation fixed, where the WIMP wind is supposed to come from. NEWSdm detector, located in the Gran Sasso Underground Laboratory, is made of a new kind of nuclear emulsions, called NIT or U-NIT (Nano Imaging Trackers or Ultra Nano Imaging Trackers) [7], with $\mathrm{AgBr}$ crystals of nanometric size immersed in an organic gelatine. The passage of 
a charged particle through the emulsion produces along its path atomic scale perturbations, called latent images. The chemical treatment makes Ag grains visible with an optical microscope. A track will therefore result as a sequence of aligned grains. NIT emulsion films make the reconstruction of trajectories with path lengths shorter than $50 \mathrm{~nm}$ possible if analyzed by means of microscopes with enough resolution. According to the equation 2.1 the total number of expected events in the whole detector is $\sim 0.30 \operatorname{ton}^{-1} \mathrm{y}^{-1}$.

\subsection{Readout strategy}

In order to detect tracks comparable and shorter than the optical resolution a two steps strategy is adopted: the first one consists of an elliptical fit for all clusters, since the scanning with optical microscope cannot distinguish two grains closer than $200 \mathrm{~nm}$. Indeed, two grains would appear as a single cluster with an elliptical shape, with the major axis along the actual direction of the recoiled nucleus, unlike a single grains from thermal excitation that would appear as spherical [8]. The first step is followed by a validation of the selected tracks with polarized light analysis using the resonance effect of nanometric metallic grains in a dielectric medium [9]. The resonance effect is sensitive to the shape of nanometric grains: when silver grains are not spherical, the resonant response depends on the polarization of the incident light. This technique provides an accuracy in the grain position of about $10 \mathrm{~nm}$ in both coordinates (see fig. 1).
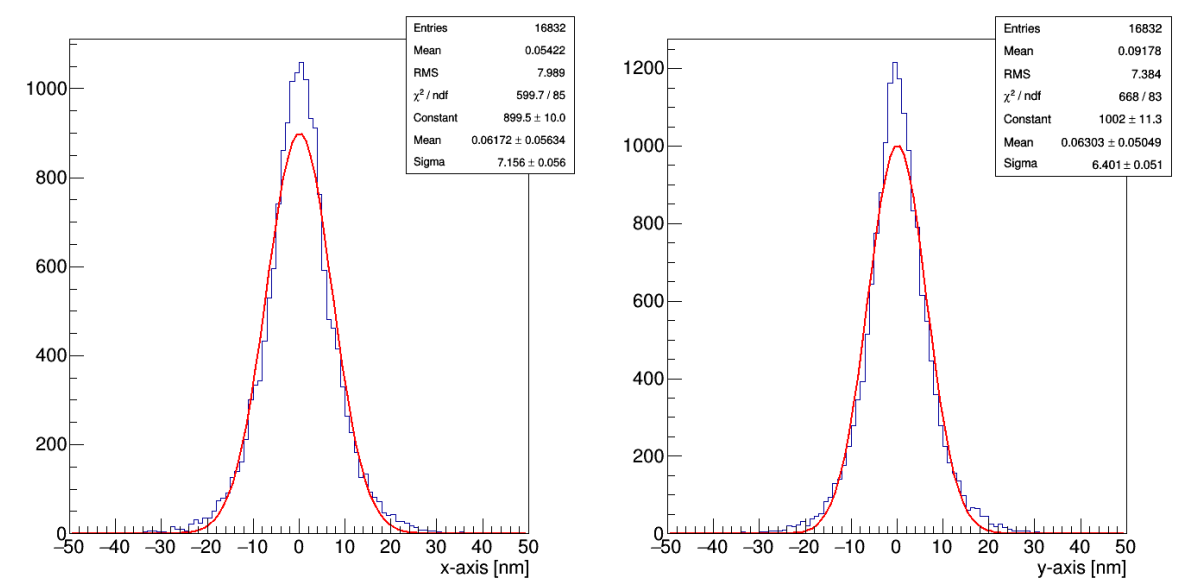

Figure 1: Position accuracy of $\mathrm{x}$ (left) and y (right) coordinates of about $10 \mathrm{~nm}$ with the resonant light scattering.

\section{Supernova neutrinos detection}

A study was performed to evaluate the capability of the NEWSdm detector to observe supernova neutrinos. In order to discriminate the signal (CEvNS interactions from supernova explosion) from the background (radiogenic neutrons [10] and solar neutrinos from ${ }^{8} \mathrm{~B}$ ) three kinematical variables were used: track length, the inclined angle of the induced recoils from the emulsion plane $\theta$ and its projection $\phi$. A likelihood ratio test A likelihood ratio test was used to estimate the significance of the test statistics $q=2 \ln \left(\mathscr{L}_{s+b} / \mathscr{L}_{b}\right)$ as a function of the exposure time for the signal plus 
background hypothesis. The shorter the exposure time the larger the significance of S+B hypothesis, since only the background increases with the time. Assuming an exposure shorter than four years, the observation of supernova neutrinos can be claimed with a significance larger than $3 \sigma \mathrm{CL}$ with a 30 ton of active detector.

\section{Conclusions}

The new generation of ton-scale detector for dark matter search are sensitive to neutrinos coming from supernova explosions. A directional detector for dark matter search, like NEWSdm, can discriminate the supernova neutrino events from the background coming mainly from radiogenic neutrons and solar neutrinos from ${ }^{8} \mathrm{~B}$ observing the angular distribution of induced recoils. A likelihood ratio test has been performed in order to evaluate the significance for the signal (supernova neutrinos) plus background (solar neutrinos from ${ }^{8} \mathrm{~B}$ and neutrons) hypothesis. A significance larger than $3 \sigma$ is obtained with 30 ton detector and exposures shorter than four years.

\section{References}

[1] H.-T. Janka. Neutrinos from type-II supernovae and the neutrino-driven supernova mechanism. In F. Giovannelli and G. Mannocchi, editors, Frontier Objects in Astrophysics and Particle Physics, page 345, 1993.

[2] M. Biassoni and C. Martinez. Study of supernova-nucleus coherent scattering interactions. Astroparticle Physics, 36(1):151 - 155, 2012.

[3] Robert Buras-Schnell, Markus Rampp, H-Th Janka, and K Kifonidis. Improved models of stellar core collapse and still no explosions: What is missing? 90:241101, 072003.

[4] T. Totani, K. Sato, H. E. Dalhed, and J. R. Wilson. Future detection of supernova neutrino burst and explosion mechanism. The Astrophysical Journal, 496(1):216, 1998.

[5] Daniel Z. Freedman. Coherent neutrino nucleus scattering as a probe of the weak neutral current. Phys. Rev., D9:1389-1392, 1974.

[6] A. Alexandrov et al. NEWS: Nuclear Emulsions for WIMP Search. LNGS-LOI 48/15. astro-ph:1604.04199, 2016.

[7] Takashi Asada, Tatsuhiro Naka, Ken-ichi Kuwabara, and Masahiro Yoshimoto. The development of a super-fine-grained nuclear emulsion. PTEP, 2017(6):063H01, 2017.

[8] M. Kimura and T. Naka. Submicron track readout in fine-grained nuclear emulsions using optical microscopy. Nuclear Instruments and Methods in Physics Research Section A: Accelerators, Spectrometers, Detectors and Associated Equipment, 680:12 - 17, 2012.

[9] Hiroharu Tamaru, Hitoshi Kuwata, Hideki T. Miyazaki, and Kenjiro Miyano. Resonant light scattering from individual ag nanoparticles and particle pairs. Applied Physics Letters, 80(10):1826-1828, 2002.

[10] A. Alexandrov et al. Intrinsic neutron background of nuclear emulsions for directional Dark Matter searches. Astropart. Phys., 80:16-21, 2016. 